\title{
Progression and treatment of incident lower urinary tract symptoms (LUTS) among men in the California Men's Health Study
}

\author{
Lauren P. Wallner, Jeff M. Slezak*, Ronald K. Loo ${ }^{\dagger}$, Virginia P. Quinn*, \\ Stephen K. Van Den Eeden ${ }^{\ddagger}$ and Steven J. Jacobsen* \\ Department of Medicine and Comprehensive Cancer Center, University of Michigan, Ann Arbor, MI, *Department of \\ Research and Evaluation, Kaiser Permanente Southern California, ${ }^{\dagger}$ Department of Urology, Southern California \\ Permanente Medical Group, Pasadena, CA, and ${ }^{\ddagger}$ Division of Research, Kaiser Permanente Northern California, \\ Oakland, CA, USA
}

\section{Objectives}

To characterise the progression and treatment of lower urinary tract symptoms (LUTS) among men aged 45-69 years in the California Men's Health Study.

\section{Patients and Methods}

A total of 39222 men, aged 45-69 years, enrolled in the Southern California Kaiser Permanente Health Plan were surveyed in 2002-2003 and again in 2006-2007. Those men who completed both surveys who did not have a diagnosis of benign prostatic hyperplasia (BPH) and were not on medication for LUTS at baseline were included in the study $(N=19505)$. Among the men with no or mild symptoms at baseline, the incidence of moderate/severe LUTS (American Urological Association Symptom Index [AUASI] score $\geq 8$ ) and odds of progression to severe LUTS (AUASI score $\geq 20$ ) was estimated during 4 years of follow-up.

\section{Results}

Of the 9640 men who reported no/mild LUTS at baseline, 3993 (41\%) reported moderate/severe symptoms at follow-up and experienced a 4-point change in AUASI score on average. Of these men, $351(8.8 \%)$ had received a pharmacological treatment, eight $(0.2 \%)$ had undergone a minimally invasive or surgical procedure and 3634 (91.0\%) had no treatment recorded. Men who progressed to severe symptoms (AUASI score $\geq 20 ; n=165$ ) were more likely to be on medication for $\mathrm{BPH}$ (odds ratio [OR] 8.09, 95\% confidence interval [CI] 5.77-11.35), have a $\mathrm{BPH}$ diagnosis (OR 4.74, 95\% CI $3.40-6.61$ ) or have seen a urologist (OR 2.49, 95\% CI 1.81-3.43) when compared with men who did not progress to severe symptoms (AUASI score $<20$ ).

\section{Conclusion}

These data show that the majority of men who experienced progression did not have pharmacological or surgical therapy for their symptoms and, therefore, may prove to be good candidates for a self-management plan.

\section{Keywords}

lower urinary tract symptoms, benign prostatic hyperplasia, progression, incidence

\section{Introduction}

Lower urinary tract symptoms are a common and burdensome condition affecting aging men. It is estimated that 3.4 million men in the USA aged $>60$ years have this condition $[1,2]$. Not only are LUTS common, but they are also associated with significant bother and poor quality of life $[3,4]$. Each year, $>\$ 6$ bn is spent on the diagnosis and treatment of LUTS [5].

In men, LUTS most often occur secondary to BPH or after prostate cancer treatment. Accurate characterisation of LUTS from the literature is difficult because of the reliance on self-report, the dynamic nature of the condition, the existence of distinct clinical subtypes of LUTS, and the various time periods over which symptoms are queried [6]. Previous studies suggest that $\sim 11-34 \%$ of men report experiencing any LUTS in their lifetime, and the prevalence of symptoms increases with age [6]; however, the majority of studies to date provide estimates of the prevalence of LUTS and cannot estimate incidence. The accurate estimation of the incidence of LUTS would further our understanding of the natural history of LUTS and, thus, facilitate research focused on prevention and improved treatment strategies. 
To date, reports on the incidence and progression of LUTS come from highly selected clinical trial populations or population-based samples of mostly white and elderly men and are therefore limited by their lack of diversity and limited availability of treatment and utilisation information over time. Results from the population-based studies suggest that between a quarter and three-quarters of men experience progression within 5 years, which strongly correlates with age [7]. Data from the Olmsted County Study (OCS) suggest that $22 \%$ of men with no/mild symptoms at baseline experienced new-onset moderate/severe LUTS within 4 years [8]. In the Osteoporotic Fractures in Men study (MrOS), up to 29\% of men with none/mild LUTS at baseline experienced clinically significant symptom progression within 2 years [9]. Martin et al. [10] found that three-quarters of Australian men in a population-based cohort experienced progression of symptoms over 5 years of follow-up. Data from the Health Professional Follow-up Study suggest that both incidence and progression increase sharply with age [7]. Data from clinical trials evaluating the effect of the pharmaceutical management of BPH/LUTS progression further support the notion that LUTS is a progressive condition, as clinically relevant symptom progression occurred in up to $20 \%$ of men with moderate/severe LUTS in the placebo arms [11-13].

To better develop strategies to manage and treat LUTS, accurate characterisation of the incidence and progression of LUTS, as well as a better understanding of treatment patterns in general practice settings is necessary; therefore, the aim of the present study was to determine the incidence of new-onset LUTS while characterising the treatment and progression of these symptoms over 4 years of follow-up among men enrolled in the large, multi-ethnic California Men's Health Study.

\section{Patients and Methods}

\section{California Men's Health Study}

The California Men's Health Study is a multi-ethnic, prospective cohort study for which recruitment, methods and population have been described previously [14-16]. Briefly, men aged 45-69 years, who were members of Northern or Southern California Kaiser Permanente Health Plans for at least 1 year before the study were eligible for inclusion. Eligible participants received questionnaires by post at baseline to ascertain demographic information, medical, family and social histories, and current health status and lifestyle behaviours. A two-stage sampling design was used for questionnaire mailing, and questionnaire data were linked to clinical data, including laboratory, hospitalisation and cancer data from electronic health plan files.

\section{Measurements}

For the present study, men from Southern California who completed both the baseline survey in 2002-2003 and the follow-up survey in 2006-2007 were included ( $n=25229)$. Of these men, those who were not continuous members of Southern California Kaiser Permanente Health Plan between surveys were excluded $(n=1506)$. Because we were interested in new-onset LUTS, we excluded men with a previous diagnosis of $\mathrm{BPH})$ before baseline $(n=2733)$, those being treated for BPH with medications before baseline $(n=1476)$ and men who had undergone a procedure to treat $\mathrm{BPH}$ before baseline $(n=9)$. As a result, a total of 19505 men were included in the present analysis.

Demographic characteristics, including age, race, education, household income and marital status, and lifestyle characteristics, e.g. smoking status, were obtained from the baseline questionnaire. Body mass index was calculated via self-reported height and weight.

The presence of LUTS was assessed at baseline and follow-up via the AUA Symptom Index (AUASI). The AUASI is a seven-item self-administered questionnaire that assesses the frequency of LUTS over the preceding month [17]. Symptom frequency questions are measured on a six-point scale from 0 (never) to 5 (always) and summed for a total AUASI score. Scores $0-7$ are considered indicative of no/mild symptoms, scores 8-19 of moderate symptoms and scores $\geq 20$ of severe symptoms. A 4-point increase in AUASI score was considered to indicate clinically relevant progression $[11,13,18]$. Information regarding BPH diagnosis (International Classification of Diseases code: ICD-9 600.x) and clinic visits to urology were ascertained from electronic medical records. Treatment for BPH/LUTS during this time was abstracted from electronic medical records and included any use of $\alpha$-blockers, $5-\alpha$ reductase inhibitors or their combination and/or the use of any minimally invasive or surgical procedure to treat BPH/LUTS (prostatectomy, transurethral resection, laser-based or microwave procedures and needle ablation).

\section{Statistical Analyses}

The distribution of demographic, lifestyle and medical characteristics was compared over levels of baseline AUASI score using chi-squared tests and $t$-tests where appropriate. The distribution of AUASI scores at follow-up was compared across levels of baseline AUASI score. Also, the distribution of the change in AUASI score from baseline to follow-up, categorised as a $<4$-point change, a 4-point increase (clinical progression) and a 4-point decrease) was compared across baseline AUASI categories. The 4-year progression of LUTS was determined among men with no/mild symptoms at baseline, as was the proportion of these men who received treatment during follow-up.

Among men with a baseline score of $0-7$, indicating no/mild symptoms, the distribution of $\mathrm{BPH}$ clinical characteristics, including a diagnosis of $\mathrm{BPH}$, use of $\mathrm{BPH}$ medication or 
Table 1 Demographic and clinical characteristics by patient AUA Symptom Index score at baseline in $2002(N=19401)^{*}$.

\begin{tabular}{|c|c|c|c|c|}
\hline & \multicolumn{4}{|c|}{2002 AUASI score } \\
\hline & $\begin{array}{c}0-7 \\
n=9701)\end{array}$ & $\begin{array}{c}8-19 \\
n=8974\end{array}$ & $\begin{array}{c}\geq 20 \\
n=726\end{array}$ & $\boldsymbol{P}$ \\
\hline Age, $n(\%)$ & & & & $<0.0001$ \\
\hline$<45$ years & $6(0.1)$ & $5(0.1)$ & $1(0.1)$ & \\
\hline $45-50$ years & $1724(17.8)$ & $1128(12.6)$ & $65(9)$ & \\
\hline $50-55$ years & $2163(22.3)$ & $1736(19.3)$ & $122(16.8)$ & \\
\hline $55-60$ years & $2289(23.6)$ & $2069(23.1)$ & $165(22.7)$ & \\
\hline $60-65$ years & $1965(20.3)$ & $2054(22.9)$ & $185(25.5)$ & \\
\hline$\geq 65$ years & $1554(16)$ & $1982(22.1)$ & $188(25.9)$ & \\
\hline Race, $n(\%)$ & & & & $<0.0001$ \\
\hline White & $6300(65.2)$ & $5800(64.9)$ & $446(61.7)$ & \\
\hline Black & $736(7.6)$ & $809(9)$ & $47(6.5)$ & \\
\hline Asian & $747(7.7)$ & $606(6.8)$ & $59(8.2)$ & \\
\hline Hispanic & $1563(16.2)$ & $1452(16.2)$ & $149(20.6)$ & \\
\hline Other & $318(3.3)$ & $273(3.1)$ & $22(3)$ & \\
\hline Unknown & $37(0.4)$ & $34(0.4)$ & $3(0.4)$ & \\
\hline Smoking status, $n$ (\%) & & & & $<0.0001$ \\
\hline Never & $4613(47.6)$ & $3936(43.9)$ & $294(40.6)$ & \\
\hline Current & $1009(10.4)$ & $1003(11.2)$ & $93(12.8)$ & \\
\hline Stopped $<6$ years ago & $4075(42)$ & $4030(44.9)$ & $338(46.6)$ & \\
\hline Unknown & $4(0.04)$ & $5(0.06)$ & $1(0.13)$ & \\
\hline Education level, $n$ (\%) & & & & $<0.0001$ \\
\hline High school or lower & $1611(16.8)$ & $1431(16.1)$ & $157(21.9)$ & \\
\hline Vocational or some college & $3320(34.5)$ & $3079(34.6)$ & $264(36.9)$ & \\
\hline College or higher & $4685(48.7)$ & $4394(49.3)$ & $295(41.2)$ & \\
\hline Unknown & $85(0.9)$ & $70(0.8)$ & $10(1.3)$ & \\
\hline Body mass index, $\mathrm{kg} / \mathrm{m}^{2}$ & & & & 0.015 \\
\hline Mean (SD) & $27.6(4.51)$ & $27.9(4.84)$ & $28.2(5.68)$ & \\
\hline Median & 27 & 27.2 & 27.3 & \\
\hline Q1, Q3 & $24.6,29.9$ & $24.7,30.2$ & $24.7,30.3$ & \\
\hline Range & $(10.8-84.1)$ & $(10.2-80.0)$ & $(7.5-67.2)$ & \\
\hline BPH medication 2002-2006, $n$ (\%) & $580(6)$ & $1217(13.6)$ & $233(32.1)$ & $<0.0001$ \\
\hline BPH diagnosis 2002-2006, $n(\%)$ & $942(9.7)$ & $1695(18.9)$ & $276(38)$ & $<0.0001$ \\
\hline BPH procedure $2002-2006, n(\%)$ & $26(0.3)$ & $46(0.5)$ & $18(2.5)$ & $<0.0001$ \\
\hline Urology visit 2002-2006, $n(\%)$ & 1840 (19) & $2205(24.6)$ & $279(38.4)$ & $<0.0001$ \\
\hline
\end{tabular}

procedure and urology clinic visits, were compared across levels of follow-up AUASI score. The associations of these $\mathrm{BPH}$ clinical characteristics with the progression to severe LUTS symptoms (AUASI score $\geq 20$ ) were estimated using multivariable logistic regression models adjusted for $\mathrm{BPH}$ medication, $\mathrm{BPH}$ procedure, urology clinic visits, $\mathrm{BPH}$ diagnosis and age. An $\alpha$ level of 0.05 was used to determine statistical significance and all analyses were performed using SAS 9.2 (Cary, NC, USA).

\section{Results}

Table 1 shows the distribution of baseline demographic and $\mathrm{BPH}$ characteristics by baseline AUASI category. Baseline symptom score was positively associated with age; a greater proportion of older men reported moderate/severe symptoms $(P<0.001)$. Men who reported no/mild symptoms at baseline were slightly more likely to be white, report that they were never smokers and have a slightly lower body mass index on average (Table 1). A diagnosis of BPH, the use of BPH/LUTS medication, a history of a procedure to treat BPH/LUTS and urology visits were all more common among men who reported severe symptoms at baseline.

Figure 1 shows the distribution of LUTS symptoms at follow-up and the change in AUASI scores between baseline and follow-up, stratified by baseline AUASI score category. Of the 9701 men who reported no/mild symptoms at baseline, $>40 \%$ reported a progression in their symptoms, with 3828 (39.7\%) reporting moderate symptoms and 165 (1.7\%) reporting severe symptoms at follow-up 4 years later. Of the 8974 men who reported moderate symptoms at baseline, the majority did not progress, again reporting moderate symptoms at follow-up (70.6\%). Among men with severe symptoms at baseline, $43.3 \%$ reported moderate symptoms and $47.9 \%$ reported severe symptoms at follow-up.

Among men with no/mild symptoms at baseline, $40.6 \%$ experienced a $\geq 4$-point increase in AUASI score over the 
Fig. 1 Follow-up AUA Symptom Index (AUASI) scores and change in AUASI scores by baseline AUASI scores $(N=19401)$.

A Follow-up AUASI category

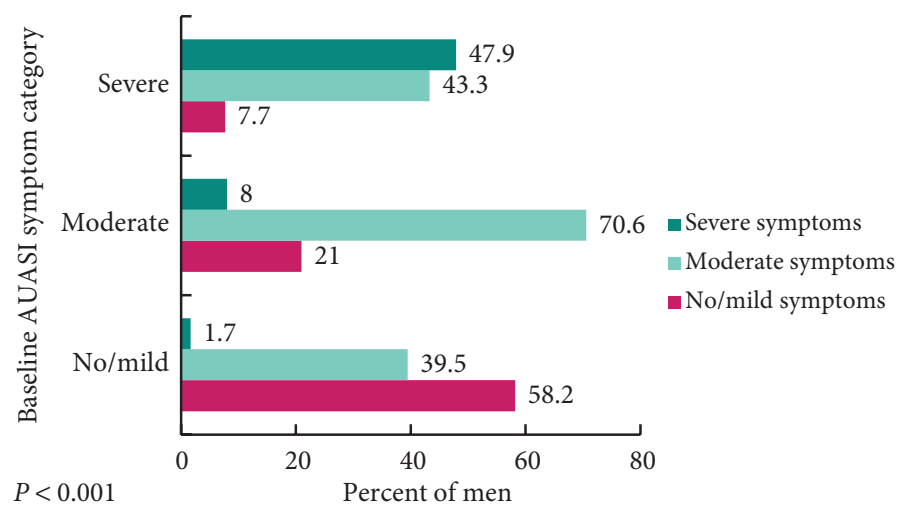

B Change in AUASI score

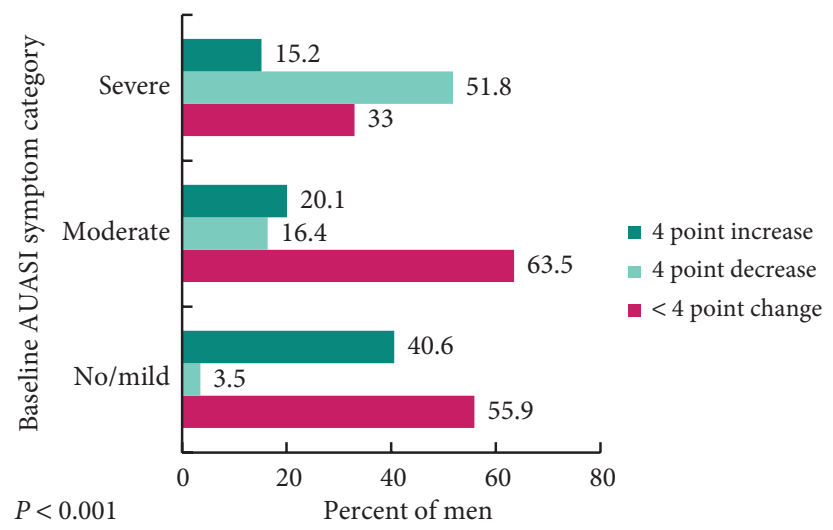

follow-up, while $55.9 \%$ experienced a $<4$-point increase or decrease and 3.5\% reported a 4 -point decrease in AUASI score. The majority of those reporting moderate symptoms at baseline experienced $\mathrm{a} \geq 4$-point decrease in AUASI score during follow-up (63.5\%), whereas $20.1 \%$ reported a $\geq 4$-point increase in AUASI score. Among men who reported severe symptoms at baseline, approximately one half experienced a 4-point decrease in AUASI score (51.8\%), a third (33.0\%) experienced a $<4$-point change and $15.2 \%$ experienced a $\geq 4$-point increase in AUASI score during follow-up (Fig. 1).

Table 2 shows the distribution of BPH clinical characteristics among the 9640 men who reported no/mild symptoms at baseline stratified by follow-up AUASI categories. Overall, the use of services during follow-up to monitor and/or treat $\mathrm{BPH}$ was positively associated with the severity of symptoms at follow-up. Men who reported severe symptoms at follow-up were more likely to have a history of $\mathrm{BPH}$ diagnosis, $\mathrm{BPH}$ medication use, $\mathrm{BPH}$ procedure use and urology visits during follow-up (Table 2). Among the 3993 men who progressed to moderate or severe symptoms, only 351 men $(8.8 \%)$ had a record of a pharmacological treatment, eight men $(2.0 \%)$ had undergone a minimally invasive or surgical procedure and 3634 men (91.0\%) had no treatment for BPH during follow-up (Table 2).

Among men with no/mild symptoms at baseline, $\mathrm{BPH}$ medication use, $\mathrm{BPH}$ diagnosis and urology visits were significantly associated with progression to severe symptoms over 4 years of follow-up. Those who progressed to severe symptoms were 4.96 times more likely to have used $\mathrm{BPH}$ medications (adjusted odds ratio [OR] 4.96, 95\% CI 3.21-7.65), 1.87 times more likely to have a $\mathrm{BPH}$ diagnosis (adjusted OR 1.87, 95\% CI 1.21-2.90) and 1.44 times more likely to have a urology visit (adjusted OR 1.44, 95\% CI 1.01-2.06) during follow-up compared with those who did not progress to severe symptoms (Table 3 ).

\section{Discussion}

Among this prospectively-followed cohort of racially diverse men participating in the California Men's Health Study, 41\% of men reported new onset of moderate/severe LUTS (AUASI $>8$ ) during the 4 years of follow-up. Of those who reported no/mild symptoms at baseline, $>40 \%$ experienced clinical progression as defined by a $>4$-point increase in AUASI score during follow-up. Even though a significant number of the men with no/mild symptoms experienced progression to moderate/severe LUTS, the vast majority (91\%) did not receive pharmaceutical or surgical treatment therapy during follow-up.

The present results support the notion that LUTS is a dynamic condition. While a significant proportion of men experienced worsening symptoms during follow-up, a subset of men in the present study saw improvement in their symptoms over the 4-year follow-up. Improvement in symptoms was most common among men with more severe symptoms and worsening of symptoms was most prevalent among men with no/mild symptoms at baseline, which may in part be influenced by regression to the mean. Our estimates of progression among men with moderate/severe symptoms are slightly lower than reported previously in the MrOS study (15 vs $25 \%$ ). This may be attributable to the inclusion of younger men in our study sample, whereas MrOS included men aged $\geq 65$ years only. Interestingly, the proportion of men without significant urinary symptoms at baseline who experienced clinical progression in their LUTS during follow-up was greater ( 41 vs $25 \%$ ). This is probably because men in the California Men's Health Study were followed for a longer period of 4 years, whereas men in the MrOs study were followed for 2 years. Our estimates were also greater than those reported by the OCS, which found $22 \%$ of men without symptoms at baseline experienced progression to moderate/severe symptoms over 42 months [8]. The difference in estimates may be partly attributable to a difference in the proportion of men who received interim treatment which, if 
Table 2 Clinical characteristics of BPH among patients with baseline AUA Symptom Index score in 2002 of $0-7$ at 2006 follow-up $(N=9640)^{*}$.

\begin{tabular}{lccrr} 
& \multicolumn{3}{c}{ 2006 AUASI score } & P \\
\cline { 2 - 4 } & $\mathbf{0 - 7}$ & $\mathbf{8 - 1 9}$ & \multicolumn{1}{c}{$\geq \mathbf{2 0}$} \\
& $\mathbf{n = 5 6 4 7}$ & $\mathbf{n = 3 8 2 8}$ & $\boldsymbol{n = 1 6 5}$ \\
\hline BPH medication 2002-2006, $\boldsymbol{n}(\%)$ & $223(3.9)$ & $298(7.8)$ & $53(32.1)$ & $<0.0001$ \\
BPH diagnosis 2002-2006, $\boldsymbol{n}(\%)$ & $403(7.1)$ & $474(12.4)$ & $54(32.7)$ & $<0.0001$ \\
BPH procedure 2002-2006, $\boldsymbol{n}(\%)$ & $18(0.3)$ & $7(0.2)$ & $1(0.6)$ & 0.4841 \\
Urology visit 2002-2006, $\boldsymbol{n}(\%)$ & $956(16.9)$ & $812(21.2)$ & $60(36.4)$ & $<0.0001$ \\
\hline AUASI, AUA Symptom Index. ${ }^{*}$ A total of 61 subjects with unknown 2006 AUASI score were excluded. &
\end{tabular}

Table 3 Logistic regression predicting AUA Symptom Index (AUASI) scores of $\geq 20$ among patients with a baseline AUASI score of 0-7.

\begin{tabular}{lcrcr}
\hline Variable & Unadjusted OR $\mathbf{( 9 5 \%}$ Cl) & \multicolumn{1}{c}{$\boldsymbol{P}$} & Adjusted OR * (95\% Cl) & P \\
\hline Age & $1.47(1.01-2.14)$ & 0.0417 & $1.04(0.71-1.54)$ & 0.8258 \\
BPH medications & $8.09(5.77-11.35)$ & $<0.0001$ & $4.96(3.21-7.65)$ & $<0.0001$ \\
BPH diagnosis & $4.74(3.40-6.61)$ & $<0.0001$ & $1.87(1.21-2.90)$ & 0.0048 \\
BPH procedure & $2.33(0.31-17.23)$ & 0.4086 & $0.42(0.05-3.22)$ & 0.4033 \\
Urology visits & $2.49(1.81-3.43)$ & $<0.0001$ & $1.44(1.01-2.06)$ & 0.0449 \\
\hline & & &
\end{tabular}

effective, would reduce LUTS over time. Also, the results may differ because of the inclusion of racial minority groups in our sample, whereas the OCS included a white-only population. Differences in both the severity and prevalence of LUTS are thought to exist across racial/ethnic groups with black and Hispanic men reporting a greater prevalence of symptoms as well as more severe symptoms compared with white men $[19,20]$.

The severity of LUTS and the odds of progression to severe symptoms were both associated with the use of services to monitor and/or treat BPH/LUTS in the present study. Men with severe symptoms at baseline were more likely to receive a clinical diagnosis of $\mathrm{BPH}$, and have been treated for BPH/LUTS during follow-up. Of those with no/mild symptoms at baseline, men who experienced progression to severe symptoms within 4 years were more likely to have treatment for BPH/LUTS, urology clinic visits and a BPH diagnosis during follow-up. Previous studies suggest bother attributable to LUTS increases with the severity of symptoms $[20,21]$ and is a strong predictor of whether or not men seek care for their LUTS [22]; therefore, it is plausible that men who have more severe symptoms are the ones seeking care for their LUTS, probably driven by the increased bother they are experiencing.

Nevertheless, the present data suggest that there remains a large population of men who experience clinically relevant progression who are not receiving care for their symptoms. This is particularly true among men with new-onset LUTS, as the vast majority of men who did not have significant
LUTS at baseline but reported moderate/severe LUTS at 4-year follow-up had not had a clinic visit nor received either drug or surgical therapy for their symptoms. The MrOS study reported a similar phenomenon, as $94 \%$ of men without significant LUTS at baseline remained untreated during the 2 years of follow-up [9]. These results confirm findings from previous studies that suggest the majority of men do not seek care for their LUTS $[3,23,24]$. Barriers to seeking care for LUTS include embarrassment about discussing symptoms, thoughts that it is a normal part of aging and lack of information regarding treatment options [25-27]. Efforts should be made to promote the discussion of LUTS during the clinic visit.

The present results suggest that a sizeable population of men experience new-onset moderate/severe LUTS within 4 years and therefore may be appropriate candidates for a self-management programme to manage their symptoms and/or prolong the initiation of drug or surgical therapy. Emberton et al. tested a self-management programme and found it to be as effective in reducing symptom progression as the standard of care (pharmaceutical management) $[28,29]$; however, despite these findings, the consensus that self-management programmes are effective in reducing LUTS and the large population of men who may benefit from such programmes, self-management is not often discussed in clinic visits and current guidelines do not prioritise self-management as a treatment strategy, largely because of the lack of an established curriculum for teaching the self-management of LUTS. 
While the present study used a uniquely prospectively followed, multi-ethnic cohort of men to understand the incidence and progression of LUTS, there are potential limitations to consider. The presence of participation bias cannot be excluded, as $40 \%$ of men who completed the baseline questionnaire did not complete the follow-up questionnaire. It is possible that those with more significant symptoms were more likely to complete both and to be included in the present analysis. This bias may have resulted in inflated estimates of LUTS progression over 4 years, but the present results are similar to those reported in previous large population-based cohorts [7-10]. In addition, data on bother attributable to LUTS were not available in this cohort and may have influenced the clinical management of men who were experiencing symptoms. The study has to date only included 4 years of follow-up and, therefore, does not allow conclusions to be drawn about the progression and management of LUTS over a longer period of time. An additional round of follow-up is currently being collected, however, which will allow estimates of 10 -year progression and investigation into associated risk factors in future analyses.

In conclusion, in the present multi-ethnic cohort of men aged 45-69 years, although $41 \%$ of men developed moderate/severe LUTS over 4 years of follow-up, $<10 \%$ of these men received surgical or drug therapy for their symptoms. As expected, treatment was more common among men who progressed to severe LUTS, but was still suboptimal. These results suggest there remains room for improvement in the clinical management of new-onset LUTS.

\section{Conflict of Interest}

None declared.

\section{References}

1 Litwin MS, Saigal CS, Beerbohm EM. The burden of urologic diseases in America. J Urol 2005; 173: 1065-6

2 Stothers L, Thom D, Calhoun E. Urologic diseases in America project: urinary incontinence in males-demographics and economic burden. J Urol 2005; 173: 1302-8

3 Welch G, Weinger K, Barry MJ. Quality-of-life impact of lower urinary tract symptom severity: results from the Health Professionals Follow-up Study. Urology 2002; 59: 245-50

4 Girman CJ, Epstein RS, Jacobsen SJ et al. Natural history of prostatism: impact of urinary symptoms on quality of life in 2115 randomly selected community men. Urology 1994; 44: 825-31

5 Saigal CS, Joyce G. Economic costs of benign prostatic hyperplasia in the private sector. J Urol 2005; 173: 1309-13

6 Wei JT, Calhoun E, Jacobsen SJ. Urologic diseases in America project: benign prostatic hyperplasia. J Urol 2005; 173: 1256-61

7 Platz EA, Joshu CE, Mondul AM, Peskoe SB, Willett WC, Giovannucci E. Incidence and progression of lower urinary tract symptoms in a large prospective cohort of United States men. J Urol 2012; 188: 496-501
8 Jacobsen SJ, Girman CJ, Guess HA, Rhodes T, Oesterling JE, Lieber MM. Natural history of prostatism: longitudinal changes in voiding symptoms in community dwelling men. J Urol 1996; 155: 595-600

9 Parsons JK, Wilt TJ, Wang PY, Barrett-Connor E, Bauer DC, Marshall LM. Progression of lower urinary tract symptoms in older men: a community based study. J Urol 2010; 183: 1915-20

10 Martin S, Lange K, Haren MT, Taylor AW, Wittert G. Risk factors for progression and improvement of lower urinary tract symptoms (LUTS) in a prospective cohort of men. J Urol 2013; 191: 130-7

11 McConnell JD, Roehrborn CG, Bautista OM et al. The long-term effect of doxazosin, finasteride, and combination therapy on the clinical progression of benign prostatic hyperplasia. N Engl J Med 2003; 349: 2387-98

12 Roehrborn CG. Alfuzosin $10 \mathrm{mg}$ once daily prevents overall clinical progression of benign prostatic hyperplasia but not acute urinary retention: results of a 2-year placebo-controlled study. BJU Int 2006; 97: $734-41$

13 Roehrborn CG. BPH progression: concept and key learning from MTOPS, ALTESS, COMBAT, and ALF-ONE. BJU Int 2008; 101 (Suppl. 3): $17-21$

14 Enger SM, Van den Eeden SK, Sternfeld B et al. California Men's Health Study (CMHS): a multiethnic cohort in a managed care setting. $B M C$ Public Health 2006; 6: 172

15 Flick E, Habel LA, Chan KA et al. Statin use and risk of prostate cancer in the California Men's Health Study cohort. Cancer Epidemiol Biomarkers Prev 2007; 16: 2218-25

16 Haque R, Van Den Eeden SK, Jacobsen SJ et al. Correlates of prostate-specific antigen testing in a large multiethnic cohort. Am J Manag Care 2009; 15: 793-9

17 Barry MJ, Fowler FJ Jr, O'Leary MP et al. The American Urological Association symptom index for benign prostatic hyperplasia. the Measurement Committee of the American Urological Association. J Urol 1992; 148: 1549-57; discussion 1564

18 Roehrborn CG, Siami P, Barkin J et al. The effects of dutasteride, tamsulosin and combination therapy on lower urinary tract symptoms in men with benign prostatic hyperplasia and prostatic enlargement: 2-year results from the CombAT study. J Urol 2008; 179: 616-21; discussion 621

19 Van Den Eeden SK, Shan J, Jacobsen SJ et al. Evaluating racial/ethnic disparities in lower urinary tract symptoms in men. J Urol 2012; 187: $185-9$

20 Sarma AV, Wei JT, Jacobson DJ et al. Comparison of lower urinary tract symptom severity and associated bother between community-dwelling black and white men: the Olmsted County Study of Urinary Symptoms and Health Status and the Flint Men's Health Study. Urology 2003; 61: 1086-91

21 Sarma AV, Jacobsen SJ, Girman CJ et al. Concomitant longitudinal changes in frequency of and bother from lower urinary tract symptoms in community dwelling men. J Urol 2002; 168: 1446-52

22 Sarma AV, Wallner L, Jacobsen SJ, Dunn RL, Wei JT. Health seeking behavior for lower urinary tract symptoms in black men. J Urol 2008; 180: 227-32

23 Sexton CC, Coyne KS, Kopp ZS et al. The overlap of storage, voiding and postmicturition symptoms and implications for treatment seeking in the USA, UK and Sweden: EpiLUTS. BJU Int 2009; 103 (Suppl. 3): 12-23

24 Jacobsen SJ, Guess HA, Panser L et al. A population-based study of health care-seeking behavior for treatment of urinary symptoms. the Olmsted County Study of Urinary Symptoms and Health Status among Men. Arch Fam Med 1993; 2: 729-35

25 Roberts RO, Rhodes T, Panser LA et al. Natural history of prostatism: worry and embarrassment from urinary symptoms and health care-seeking behavior. Urology 1994; 43: 621-8 
26 Shaw C. A review of the psychosocial predictors of help-seeking behaviour and impact on quality of life in people with urinary incontinence. J Clin Nurs 2001; 10: 15-24

27 Shaw C, Tansey R, Jackson C, Hyde C, Allan R. Barriers to help seeking in people with urinary symptoms. Fam Pract 2001; 18: 48-52

28 Brown CT, . Yap T, Cromwell DA et al. Self management for men with lower urinary tract symptoms: randomised controlled trial. BMJ 2007; 334: 25

29 Brown CT, van der Meulen J, Mundy AR, O'Flynn E, Emberton E. Defining the components of a self-management programme for men with uncomplicated lower urinary tract symptoms: a consensus approach. Eur Urol 2004; 46: 254-62; discussion 63
Correspondence: Lauren P. Wallner, Department of Medicine and Comprehensive Cancer Center, University of Michigan, North Campus Research Complex, 2800 Plymouth Road, Building 16, 409E, Ann Arbor, MI 48109, USA.

e-mail: lwallner@med.umich.edu

Abbreviations: AUASI, AUA Symptom Index; OR, odds ratio; MrOS, Osteoporotic Fractures in Men study. 